Volume 1, Issue 1, 2011, 031-040

Received: 8.02.2011 / Accepted: 12.02.2011 / Published on-line: 15.02.2011

\title{
In vitro methods for the study of microbial biofilms
}

\section{Crina Saviuc $^{1 *}$, Alexandru Mihai Grumezescu ${ }^{2}$, Mariana Carmen Chifiriuc ${ }^{1}$, Coralia Bleotu $^{3}$, George Stanciu ${ }^{4}$, Radu Hristu ${ }^{4}$, Dan Mihaiescu ${ }^{2}$, Veronica Lazăr ${ }^{1}$}

ABSTRACT

\begin{abstract}
The emergence of microbial biofilm related infections (bacterial and fungal) has a significant impact for the human pathology in the entire world. The understanding of microbial infections related to the biofilm development on tissues or indwelling devices was possible by using different qualitative and quantitative in vitro assays, in continuous and discontinuous systems, as well as in vivo models. A necessary step for obtaining more standardized, reliable and comparable results among different laboratories is the simplification of the available techniques used for investigating the biofilm formation and properties, including the biofilms susceptibility to antibiotics. The aim of the present study was to exemplify a series of available methods for the investigation of in vitro microbial biofilms developed on inert substrata, as well as coated with ferrite nanoparticles, using as experimental model a Sacharomyces cerevisiae strain. Microbial biofilm architecture was directly examined by two microscopy techniques (inverted microscopy and confocal laser microscopy scanning). The in vitro study of the influence of suspended ferrite nanoparticles on planktonic cells growth, adherence and consecutive biofilm development on inert substrata was performed by using a simple microtiter method.
\end{abstract}

Keywords: microbial biofilms, tolerance to antibiotics, Saccharomyces cerevisiae, core/shell nanoparticles, inverted microscopy, CLSM

\section{Introduction}

Biofilms are sessile microbial communities which are ubiquitous in natural and industrial environment. Biofilm development as a multistage process governed in terms of dynamics by various physico- chemical principles and biological processes [1]. Fungal biofilms related infections became a major cause of human disease, in fact, since 1979 in United States the annual incidence of fungal sepsis increased over $200 \%$ [2]. Invasive fungal infections result in substantial morbidity and mortality ( 0.4 deaths per 100,000 population, especially in immunocompromised patients), while medical devices became an essential component in the supportive care of many patients, the fungal infections related to biofilm development on indwelling devices being of significant importance for

\footnotetext{
${ }^{1}$ University of Bucharest, Faculty of Biology, Microbiology University Department

*Corresponding author e-mail address: crina.saviuc@yahoo.com

${ }^{2}$ University Politechnica of Bucharest, Faculty of Applied Chemistry and Materials Science, Organic Chemistry Department

${ }^{3}$ Institute of Virology, St. Nicolau Bucharest, Romania

${ }^{4}$ University Politechnica of Bucharest, Faculty of Applied Science, Center for Microscopy - Microanalysis and Information Processing
} 
the human pathology $[3,4]$. The pathogenesis of invasive fungal infections depends on the type and properties of the microorganisms included into the biofilm and is facilitated by a number of virulence factors, the most important being the ability to adhere to the host cells and to form biofilms characterized by a higher resistance to antifungal agents comparatively to the planktonic cells, as well as the secretion of hydrolytic enzymes, such as proteases, phospholipases and lipases $[4,5,6]$.

The adherence to abiotic substrata is influenced by several factors correlated with the substrate nature (hydrophobicity, surface energy and other physico-chemical properties), the presence of a conditioning film and with the microenvironment chemical composition $[7,8,9,10]$. Furthermore, since the process of microbial adhesion to indwelling medical devices is passing in the presence of body fluids flow, physical forces such as shear generated by local hemodynamics may modulate the adhesion process [7]. The investigation of biofilm formation in all steps, from the initial adherence to the resulting mature biofilm, and of the special phenotype of microorganisms included in such communities, exhibiting high resistance to antimicrobial agents sometimes called tolerance or recalcitrance, is a must in order to improve the therapeutic approach of biofilm associated chronic infections [8]. The understanding of these processes was possible by using different qualitative and quantitative assays in vitro, in continuous and discontinuous systems, as well as in vivo models. The thermodynamic approach of surface properties both of substratum and microbial cell wall according to DLVO theory (named after Derjaguin and Landau, Verwey and Overbeek) was studied in order to establish possible interactions between complementary structures.

In clinical microbiology laboratories several methods are used for recovering and measuring the biofilms formed on medical devices, especially coupled with viable cell counts, after dispersion of adhered cells [8]. Traditionally, the majority of experimental models used for the development of artificial microbial biofilms, including those formed by fungal species, are requiring expert handling, longer processing times and the use of specialized equipment not generally available in a regular microbiology laboratory. The experimental substrata used by different groups of investigators are very diverse, including catheter sections, sheets and tubing from medical devices, glass and plastic slides, cylindrical cellulose filters, acrylic strips and discs, germanium substratum, tissue culture flasks, syringes, and the methods are including both static and flow-through systems (e.g. modified Robbins devices, Calgary biofilm device, the CDC biofilm reactor, fermentors, etc.). In this context, colorimetric assays of cellular viability and biomass quantification by staining techniques (crystal violet staining technique), seem to be simple and valuable solutions [6,11]. A mainstay of colorimetric assays uses tetrazolium salts, i.e. the XTT model based on the ability of metabolically active sessile cells to reduce a tetrazolium salt to water-soluble orange formazan compounds, whose intensity can be determined using a microtiter-plate reader. The technique has limitations especially due to low possibility of inter-strains comparisons in the absence of detailed standardization, since different strains metabolize the substrate with different capabilities, and the amount of intracellular retained product may vary between different cellular states, e.g., planktonic and sessile [12].

Biofilm detection and study are facilitated by microscopy techniques. Confocal scanning laser microscopy (CLSM) is a three dimensional method working both in reflexion and transmission mode, used for the in situ study of microbial associations with different surfaces. Digital imaging methods can be used to create computer reconstructions of the chemical and physical conditions within the micro-environments of microbial communities. Scanning electron microscopy (SEM) is an important tool, providing high resolution visualization of microbial biofilms. In SEM, biofilm specimens are prepared by fixation, staining, drying and conductively coating prior to imaging under 
high vacuum. While any pretreatment can alter specimen morphology, drying appears to significantly impair biofilms due to exopolymeric substance (EPS) collapsing. Several techniques could reduce the drying effect, as wet-mode environmental scanning electron microscopy (ESEM), which is examining fresh, hydrated specimens, preventing the drying by working in a gaseous atmosphere, compared with SEM requiring vacuum conditions [13]. Although similar preparation is used in transmission electron microscopy (TEM), specimens for TEM are embedded in a resin which physically stabilizes the EPS matrix. However, TEM is not applicable for observing the extent and surface of biofilms, which is often of interest. Last but not least, mathematical models integrating experimental results can predict the complex process of biofilm formation dynamic [13]. The development of the biofilm mathematical models has undergone an evolution from simple to complex, from one spatial dimensional models to multidimensional models, from single species to multispecies models, from steady-state models to dynamic ones, from pure growth to models including biomass growth and biofilm-fluid interaction, from describing a particular process to incorporating many different processes occurring simultaneously, from qualitative to quantitative, from single scale to multiscale ones etc [1]. Important issues as AFM (atomic force microscopy), immunological and genetic assays are strong contributors to the knowledge of microbial biofilms [1, 6]. However, a necessary step for obtaining more standardized, reliable and comparable results among different laboratories is the simplification of the available techniques used for investigating the biofilm formation and properties, including the biofilms susceptibility to antibiotics [6]. Core/shell particles with nano-diameter are very attractive in a wide range of industrial fields, such as electronics, diagnosis and paint applications, because of their functionality and dispersion stability properties. Ferrite $\left(\mathrm{Fe}_{3} \mathrm{O}_{4}\right)$ nanoparticles are naturally hydrophilic because they are containing are a lot of hydroxyls on the surface, and thus difficult to disperse in organic media. Therefore, an organic functionalization of nanoparticles is very important for their biocompatibility. They also exhibit low cytotoxicity. The nanoparticles can either be used for simple adsorption of biomolecules, or functionalized or encapsulated in polymers or silica materials to fabricate hybrid composites with increased biocompatibility and added functionalities, like delivery mechanism for antimicrobial agents, or coating films for preventing microbial adherence [14]. The aim of the present study was to exemplify a series of available methods for the in vitro investigation of fungal biofilms developed on inert substrata, as well as coated with ferrite nanoparticles.

\section{Experimental section}

Microbial model. The artificial monospecific biofilms were developed using a Sacharomyces cerevisiae strain recently isolated from a clinical specimen (sputum) and identified by using Vitek II automatic system and previously tested for susceptibility to currently used antifungals (voriconazole, itraconazole, caspofungin B, amphotericin B, fluconazole, flucytosin) and some essential oils [15].

Inert substrata used for the microbial adherence assay. Glass cover-slips were used for the bioassays, with and without nanoparticles coating. Core/shell type nanoscale materials from 5-20nm diameter were obtained by adapted Massart method under microwave conditions [16, 17]. Subsequently, $\mathrm{Fe}_{3} \mathrm{O}_{4} /$ oleic acid nanoparticles were dispersed in chloroform, oriented in magnetic field and the cover-slips were one submerged in nanofluid and extemporaneously dried because of convenient volatility of chloroform $[18,19]$. 
Filamentation assay: The yeast strain was cultivated overnight on Sabouraud medium. From 24h culture a microbial suspension of 2 McFarland density was obtained, inoculated $1: 1 \mathrm{v} / \mathrm{v}$ in bovine serum and incubated for $2 \mathrm{~h}$ at $37^{\circ} \mathrm{C}$. The fresh preparations were microscopically examined with $40 \mathrm{x}$ objective.

Fungal growth curve. The $S$. cerevisiae strain was cultivated in liquid glucose broth, incubated at $25^{\circ} \mathrm{C}$ and spectrophotometrically assessed at different time intervals in order to draw the growing curve using the optical density values.

Experimental model for the in vitro study of biofilm development on the inert substrata (plastic 96 multi-well plates and glass coverslips) and assessment of the influence of ferrite nanoparticles on planktonic cells growth and biofilm development, using the microtiter method. In this purpose, two-fold microdilutions of nanoparticules stock solutions prepared in DMSO were performed in liquid medium distributed in 96 multi-well plates, starting from 33.3 $\mathrm{mg} / \mathrm{mL}$ to $0.0325 \mathrm{mg} / \mathrm{mL}$ ). Serial dilutions of suspension liquid (in this case DMSO) were performed in order to obtain the negative control. Each well was inoculated with $5 \mu \mathrm{L}$ of $0.5 \mathrm{Mc}$ Farland microbial suspension. Sterility control wells (glucose broth) and microbial growth controls (inoculated glucose broth) were used. The plates were incubated for $24 \mathrm{~h}$ at $30^{\circ} \mathrm{C}$, and the influence of nanoparticles on the planktonic cells growth was appreciated by measuring the A $600 \mathrm{~nm}$. Thereafter, the 96 well plates were emptied, washed 3 times with PBS, fixed with cold methanol and stained with crystal violet solution $1 \%$ for $30 \mathrm{~min}$. The biofilm formed onto the plastic wells was resuspended in $30 \%$ acetic acid and the intensity of the intensity of colored suspension was assayed by measuring the absorbance at $490 \mathrm{~nm}$ [20].

Microbial adherence assay to coated and uncoated materials. The microbial adherence ability was investigated in 6 multiwell plates, in which there have been placed coverslips with and without coating treatment. Plastic wells were filled with liquid medium, inoculated with $300 \mu \mathrm{L} 0.5$ McFarland microbial suspension and incubated for 72 hours at $30^{\circ} \mathrm{C}$. After 24 hours the culture medium was removed, cover slips were washed three times in PBS in order to remove the nonadherent free and fresh Glucose broth was added [21, 22, 23].

Direct examination of biofilm architecture by microscopy techniques. The samples were represented by uncoated coverslips and coverslips coated with core/ shell nanosystems covered by fungal biofilms. For the microbial biofilm architecture assessment, a CLSM technique was used. After 24, 48 and $72 \mathrm{~h}$ of incubation the samples were removed from the plastic wells, washed three times with phosphate buffered saline (PBS), fixed with cold methanol and dried before microscopic examination. Samples were visualized in reflection mode by using a Leica microscope (TCS-SP CSLM model), equipped with PL FLUOTAR (40X NA0.7, electronic zoom 1) and an He-Ne laser tuned on $633 \mathrm{~nm}$ wavelength. A lateral resolution of about $600 \mathrm{~nm}$ was achieved. A special Leica software was used for surface topography and for statistical analysis. Simultaneously, after 24, 48 and $72 \mathrm{~h}$ respectively, the biofilms developed on the coverslips were also examined by an inverted microscope (40X).

\section{Results section}

The Saccharomyces cerevisiae yeast model, when appropriate, draws on studies of other fungi for comparison. The Klis laboratory made many contributions to the development of the view 
that the cell walls of $C$. albicans, a major fungal pathogen and that of $S$. cerevisiae are similar in their covalent structures [24, 25]. A powerful attraction of $S$. cerevisiae is that the whole organism is under intensive study, providing information on all aspects of its biology as they relate to the cell wall. The cell surface has two essential roles: to maintain the integrity of the cell and to interact with the environment. In this context, studies on fungal biofilm formation can adopt the $S$. cerevisiae model. The tested strain isolated from sputum proved to be susceptible to all tested antifungal drugs (Fluconazole, Itraconazole, Voriconazole, Flucytosin, Amphotericin B) and to essential oils (MIC range from 0.05 to $6.75 \mu \mathrm{L} / \mathrm{mL}$ ) [15].

Using the simple assay of filamentation (yeast to hyphae switch), which can reveal fungal dimorphism, it was demonstrated that the tested strain did not exhibit the ability to form pseudohyphae after $2 \mathrm{~h}$ of incubation with bovine serum (figure 1 ).

Fig. 1. S. cerevisiae filamentation assay (x40, fresh preparation)

The results were well correlated with the microscopic examination of biofilm formed at $24 \mathrm{~h}$ on coverslips.

After $24 \mathrm{~h}$ the tested strain reached the stationary faze which lasted to $120 \mathrm{~h}$.

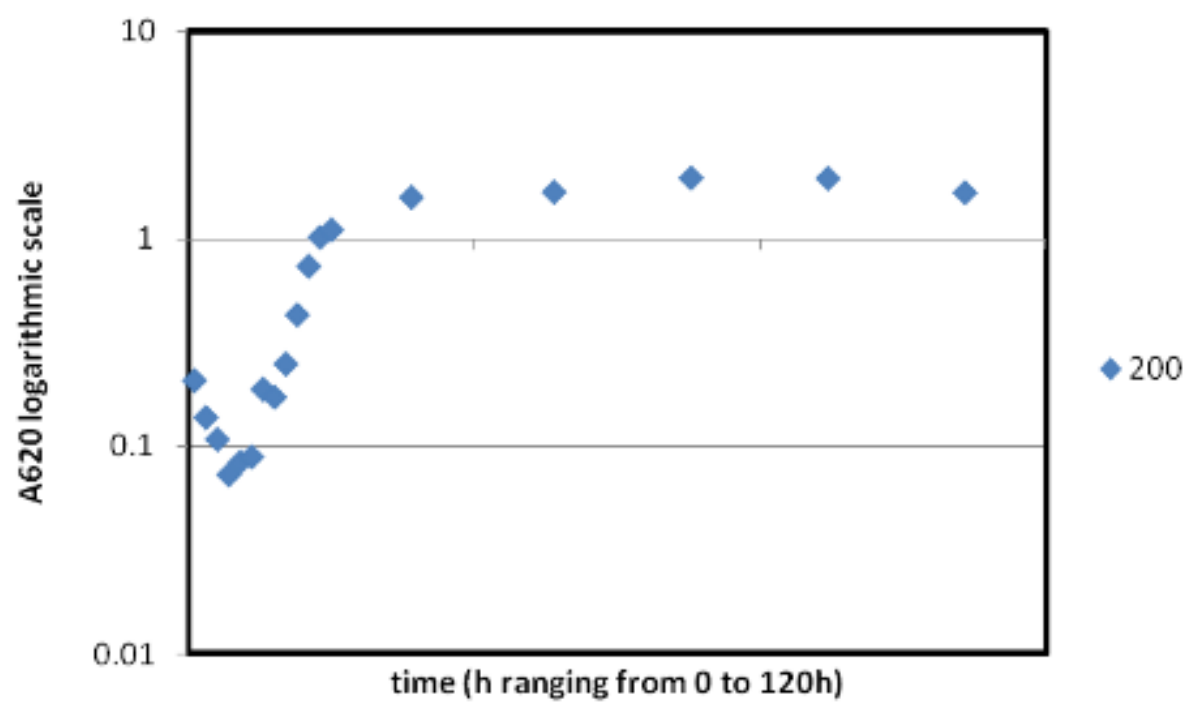

Fig. 2 Growing curve of the $S$. cerevisiae strain

A comparison between the absorbance of suspended cells (A 620nm) and adhered ones (A $490 \mathrm{~nm}$ ) was made in order to establish the antimicrobial or antibiofilm effect of core/shell ferrite nanoparticles. After $24 \mathrm{~h}$

a positive influence on the microbial growth, proportional with the concentration of the functionalized nanosystems suspension was observed, instead of the inhibitory activity of the same concentrations on the biofilm development (fig. 2). 


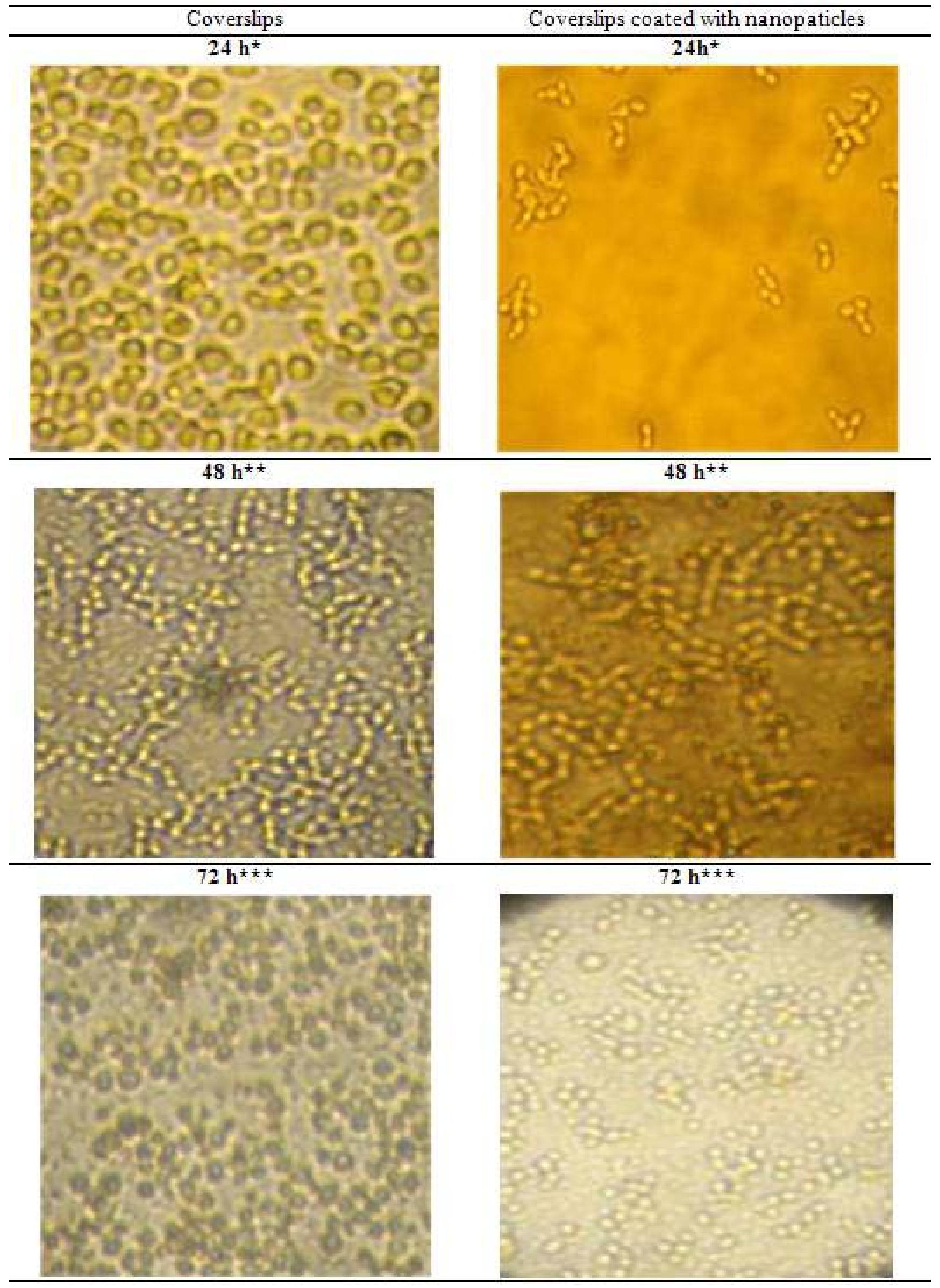




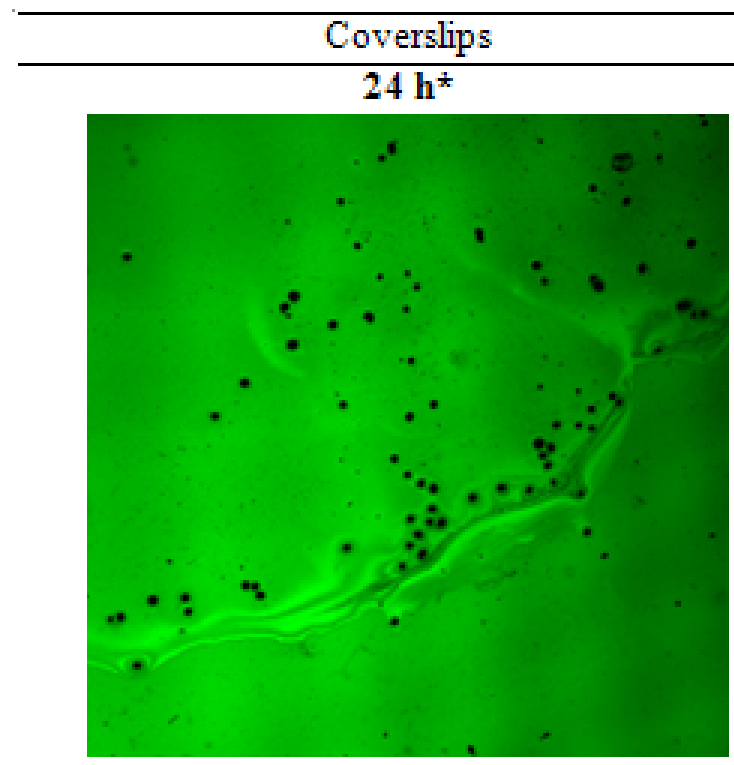

Coverslips coated with nanopaticles
$\mathbf{2 4 \mathbf { h } ^ { * }}$
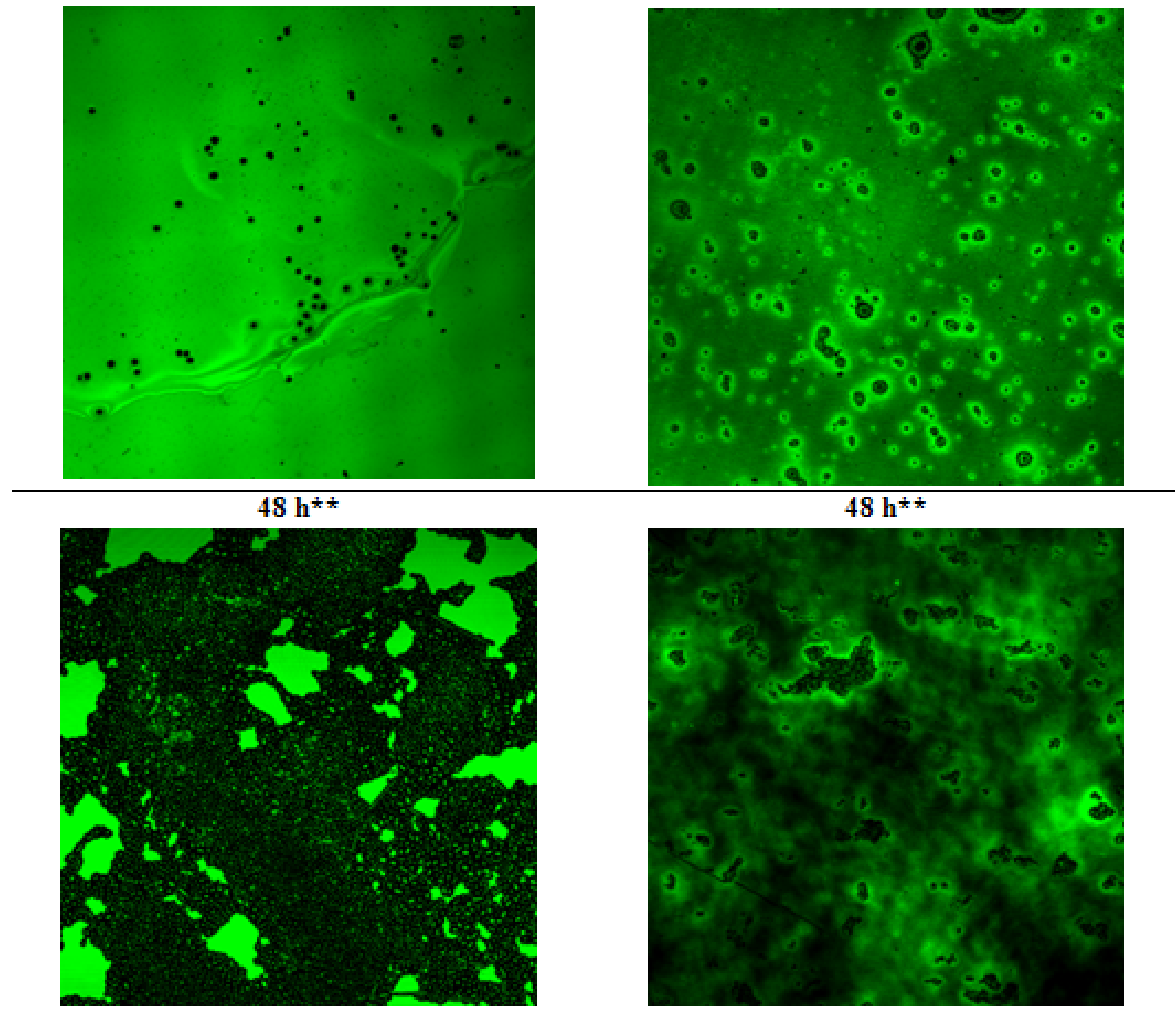

$48 h^{* *}$
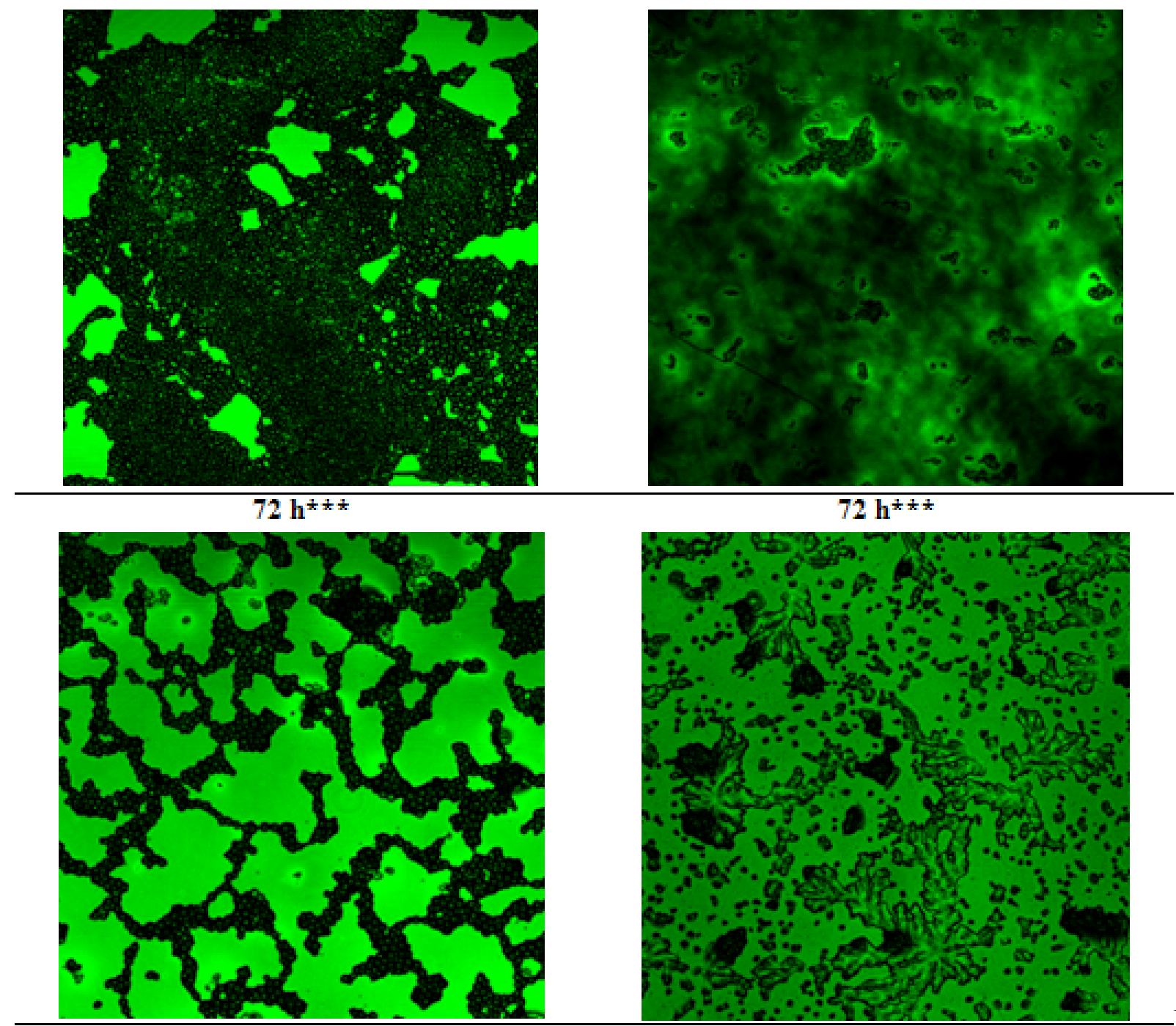

Fig.

3. Microscopic images of $S$. cerevisiae biofilms developed on cover-slips by inverted and CLSM tecniques $(250 \mu \mathrm{m} \times 250 \mu \mathrm{m})$; objective 40X, NA 0.7) 


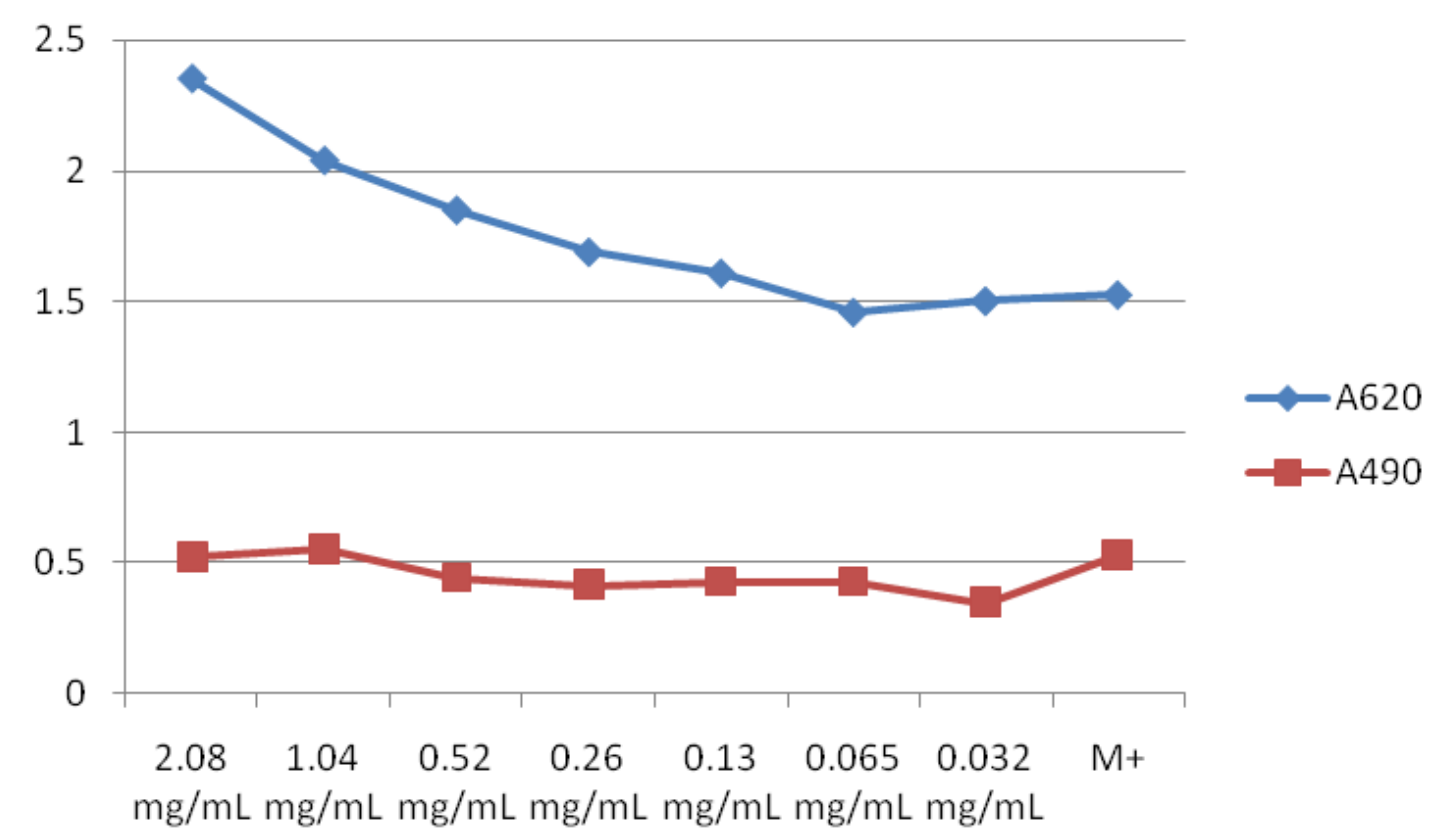

Fig. 2. Influence of ferrite corre/shell type nanoparticles on the growth of planktonic (A $620 \mathrm{~nm}$ ) /adherent (A $490 \mathrm{~nm}$ ) fungal strains

The biofilm development was appreciated based on the following features: microcolonies/biofilm presence, degree of surface coverage, the biofilm thickness, the presence of different specialized structures inside the biofilm and of protective matrix, the presence and abundance of pseudohyphae. The two microscopy techniques used for samples observation offered complementary information.

At $24 \mathrm{~h}$, CLSM revealed no adherence in case of coated and uncoated glass material (Fig. 3). The CLSM proved to be a better tool, concerning the qualitative appreciation of biofilm architecture complexity, the coverage degree on the substratum surface and the disruption of biofilm in the presence of inhibitory substances. The direct examination of the fresh biofilms by inverted microscopy provided more reliable information concerning the fungal cells morphology.

\section{Conclusions}

The understanding of microbial biofilms architecture and properties is requiring the development of accurate and realistic experimental models and concerted efforts of scientists from a variety of disciplines, able to explore these complex systems of the microbial world in order to solve the problem of biofilms associated infections, by using adequate methods for the assessment of biofilms resistance to different antimicrobial substances, and by designing new therapeutical methods and anti-pathogenic strategies based on the inhibition of the intercellular communication and virulence genes expression.

\section{References}

[1] Wanga Q., Zhang T., Review of mathematical models for biofilms, Solid State Communications, 150, 1009-1022, 2010 
[2] Trofa D., Ga'cser A., Nosanchuk J. D., Candida parapsilosis, an Emerging Fungal Pathogen, Clinical Microbiology Reviews, 606-625, 2008

[3] Andes D., Nett J., Oschel P., Albrecht R., Marchillo K., Pitula A., Development and Characterization of an In Vivo Central Venous Catheter Candida albicans Biofilm Model, Infection and Immunity, 72, 10, 6023-6031, 2004

[4] Weber K., Sohr R., Schulz B., Fleischhacker M., and Ruhnke M., Secretion of E,E-Farnesol and Biofilm Formation in Eight Different Candida Species, Antimicrobial Agents and Chemotherapy, 52, 5, 1859-1861, 2008

[5] Pierce C., Uppuluri P., Tristan A., Wormley Jr. F., Mowat E., Ramage G., Lopez-Ribot J., A simple and reproducible 96 well plate-based method for the formation of fungal biofilms and its application to antifungal susceptibility testing, Nature Protocols., 3, 9, 1494-1500, 2008

[6] Lazar V., Aderenta Microbiana, Editura Academiei Romane, 2003

[7] Katsikogianni M.G., Missirlis Y.F., Interactions of bacteria with specific biomaterial surface chemistries under flow conditions, Acta Biomaterialia, 6, 1107-1118, 2010

[8] Lazar V., Chifiriuc M. C.. Medical significance and new therapeutical strategies for biofilm associated infections. Roumanian Archive of Microbiology and Immunology, 69, 3, 121-127, 2010

[9] Chifiriuc M. C., Bleotu C., Marutescu L., Cristea D., Lazar V., The modulation of hela cells secretory patterns by invasive Shigella spp. and enteroinvasive E. coli bacterial cells and their soluble components, Roumanian Archive of Microbiology and Immunology, 69, 3, 139-145, 2010

[10] Lazar V., Chifiriuc M.C., Stewart Tull D.E.S., Bleotu C., Candlich D., Wardlaw A.C., Investigation of the cytotoxic capacity of some adherent opportunistic enterobacterial strains by the MTT and TEM, Roumanian Archive of Microbiology and Immunology, 69, 1, 35-40, 2010

[11] Saviuc C., Dascalu L., Chifiriuc M. C., Radulescu V., Oprea E., Popa M., Hristu R., Stanciu G., Lazar V., The inhibitory activity of pomelo essential oil on the bacterial biofilms development on soft contact lenses, Romanian Archives of Microbiology and Immunology, 69, 3, 140-145, 2010

[12] Kuhn D. M., Balkis M., Chandra J., Mukherjee P. K., Ghannoum M. A., Uses and Limitations of the XTT Assay in Studies of Candida Growth and Metabolism, Journal of Clinical Microbiology, 41, 1, 506508,2003

[13] Priester J., Horst A., Van De Werfhorst L., Saleta J., Mertes L., Holden P., Enhanced visualization of microbial biofilms by staining and environmental scanning electron microscopy, Journal of Microbiological Methods, 68 577-587, 2007

[14] Liang X. J., Chen C., Zhao Y., Jia L., and Wang P., Biopharmaceutics and Therapeutic Potential of Engineered Nanomaterials, Current Drug Metabolism, 9,8, 697-709, 2008

[15] Saviuc C., Grumezescu A. M., Oprea E., Radulescu V., Dascalu L., Chifiriuc M. C., Bucur M., Lazar V., Anti-fungal activity of some vegetal extracts on Candida biofilms developed on inert substratum, Biofilms in noscomila fungal infections, Paris, France, 31 jan-1-feb 2011

[16] Buteică A. S., Mihaiescu D. E., Grumezescu A. M., Vasile B. S., Popescu A., Călina D., Mihaiescu O. M., The cytotoxicity of (non)magnetic nanoparticles tested on Escherichia coli and Staphylococcus aureus, Digest Journal of Nanomaterials and Biostructures, 5, 3, 651, 2010

[17] Buteică A. S., Mihaiescu D. E., GRumezescu A. M., B. Ş. Vasile B. S., Popescu A., Mihaiescu O. M., Cristescu R., The anti-bacterial activity of magnetic nanofluid: $\mathrm{Fe}_{3} \mathrm{O}_{4}$ /oleic acid/cephalosporins core/shell/adsorptionshell proved on $S$. aureus and E. coli and possible applications as drug delivery systems, Digest Journal of Nanomaterials and Biostructures, 5, 4, 927, 2010

[18] Chifiriuc M.C., Lazar V., Bleotu C., Calugarescu I., Grumezescu A.M., Mihaiescu D. E., Mogoşanu D. E., Buteica A.S., Buteica E., Bacterial adherence to the cellular respectively inert substrate in the presence of magnetic $\mathrm{CoFe}_{2} \mathrm{O}_{4}$ and $\mathrm{Fe}_{3} \mathrm{O}_{4}$ /oleic acid - core/shell nanoparticle, Digest Journal of Nanomaterials and Biostructures, 6, 1, 37-42, 2011

[19] Grumezescu A. M., Mihaiescu D. E., Mogoşanu D. E., Chifiriuc M. C., Lazăr V., Călugărescu I., Trăistaru V., In vitro assay of the antimicrobial activity of $\mathrm{Fe}_{3} \mathrm{O}_{4}$ and $\mathrm{CoFe}_{2} \mathrm{O}_{4}$ / oleic acid - core/shell on 
clinical isolates of bacterial and fungal strains, Optoelectronics and Advanced Materials - Rapid Communications, 4, 11, 1798 - 1801, 2010

[20] Lazar V., Balotescu M. C., Cernat R., Bulai D., Nițu G., Ilina L., Phenotypic antibioticresistance of some enterobacterial strains adhered to an inert substratum. In vitro models for biofilm formation in liquid and solid media, Clinical Microbiology and Infection, 9, [Suppl], 227, 2003

[21] Herlea V., Lazar V., Canja D., Yeasts as a Model Eukariotic Cell - Biotechnological implications, Procedings of the $8^{\text {th }}$ National Symposium of Industrial Microbiology and Biotechnology, Anghel It ed., Bucharest, Romania, 103-112, 1994

[22]Lazar V., Chifiriuc M. C., Cernat R. C., Bulai D., Method for Determining Bacterial Biofilm Sensitivity to Antimicrobial Substances, RO123145-B1

[23]Lazar V., Chifiriuc M. C., Cernat R. C., Bulai D., Method for Determining The Microbial Biofilm Sensitivity to Antimicrobial Substances, RO123146-B1

[24] Lesage G., Bussey H., Cell Wall Assembly in Saccharomyces cerevisiae, Microbiology and Molecular Biology Reviews, 70, 2, 317-343, 2006

[25] LaJean Chaffin W., Candida albicans Cell Wall Proteins, Microbiology and Molecular Biology Reviews, 72, 3,495-544, 2008

Acknowledgement: This research was financed by the European Funding Program POSDRU 107/1.5/S/80765 and the Romanian Research project PN2 42150/2008-2011 